\title{
FRECUENCIA DE HEPATITIS A EN NIÑOS Y ADOLESCENTES DE CINCO CIUDADES DEL PERÚ
}

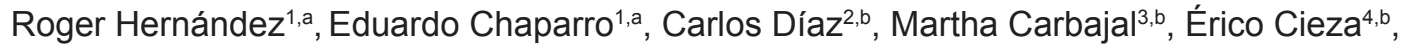 \\ Rosario Cerpa ${ }^{5, a}$
}

\begin{abstract}
RESUMEN
Con el objetivo de determinar la frecuencia de hepatitis A en niños y adolescentes en las ciudades de Lima, Arequipa, Piura, Cajamarca e Iquitos, se realizó un estudio transversal descriptivo para lo cual se midió anticuerpos antihepatitis A en suero de 1721 niños y adolescentes de 1 a 15 años. La frecuencia de serología positiva global fue de 50,5\% (IC 95\%: 48,1-52,9), siendo menor para la ciudad de Lima con el 37,4\% (IC 95\%: 35,1-39,8) y mayor para la ciudad de lquitos con el $68,7 \%$ (IC 95\%: 63,6-73,4). La seropositividad fue mayor en el grupo de 10 a 15 años con 66,1\% y en el nivel socioeconómico del grupo $\mathrm{E}$ con el $64,6 \%$. Se concluye que las ciudades estudiadas tienen un patrón de endemicidad intermedio. La frecuencia de hepatitis A fue mayor en los niños y adolescentes de menor nivel socioeconómico y de mayor edad.
\end{abstract}

Palabras clave: Hepatitis A; Niño; Adolescente; Nivel socioeconómico (fuente: DeCS BIREME).

\section{FREQUENCY OF HEPATITIS A IN CHILDREN AND ADOLESCENTS FROM FIVE CITIES OF PERU}

\begin{abstract}
In order to determine the frequency of hepatitis $\mathrm{A}$ in children and adolescents in the cities of Lima, Arequipa, Piura, Cajamarca and lquitos, a descriptive cross-sectional study which measured serum anti-hepatitis $A$ antibodies from 1,721 children and adolescents aged 1-15 years was performed. The overall frequency of positive serology was $50.5 \%(95 \% \mathrm{Cl}$ : 48.1 to 52.9$)$, with lower rates for Lima at $37.4 \%(95 \% \mathrm{Cl}: 35.1$ to 39.8$)$ and higher rates for lquitos at $68.7 \%$ (95\% Cl: 63.6 to 73.4). Seropositivity was higher in the group of $10-15$ year-olds with $66.1 \%$ and in the socioeconomic status group $E$ with $64.6 \%$. We concluded that the cities studied have a pattern of intermediate endemicity. The frequency of hepatitis A was greater in children and adolescents of lower socioeconomic status and older age.
\end{abstract}

Key words: Hepatitis A; Child; Adolescent; Social class (source: MeSH NLM).

\section{INTRODUCCIÓN}

A nivel mundial ocurren aproximadamente un millón cuatrocientos mil casos anuales de hepatitis A. Los cuales se presentan principalmente en regiones de menor desarrollo, como África, América Central y Sudamérica ${ }^{(1)}$. La hepatitis $A$ es una de las principales causas de infección trasmitida a través del agua y los alimentos. El virus de la hepatitis A (VHA) es muy resistente al medio ácido y al calor, por lo que puede sobrevivir días a semanas en agua, suelo y en los alimentos. Estas características del virus, sumadas a malas condiciones de salubridad e higiene presentes en muchas zonas de Latinoamérica, permiten que el VHA sea el agente etiológico más frecuente de las hepatitis virales ${ }^{(2-6)}$.

En regiones con alta endemicidad de hepatitis $A$ (anticuerpos antihepatitis A positivo es al $90 \%$ a los 10 años de edad), la exposición es temprana y casi toda la población está infectada; en este escenario predomina la infección asintomática, las tasas de notificación son bajas y los brotes son poco frecuentes debido a la alta prevalencia de inmunidad en la población ${ }^{(3,4)}$.

\footnotetext{
Hospital Cayetano Heredia. Lima, Perú.

Hospital EsSalud Iquitos. Iquitos, Perú.

Hospital Regional Cayetano Heredia. Piura, Perú

Hospital Regional de Cajamarca. Cajamarca, Perú.

Hospital Nacional Edgardo Rebagliati Martins. Lima, Perú.

Médico infectólogo pediatra; ${ }^{b}$ Médico pediatra

Recibido: : 29-01-15 Aprobado: 05-08-15
}

Citar como: Hernández R, Chaparro E, Díaz C, Carbajal M, Cieza E, Cerpa R. Frecuencia de hepatitis A en niños y adolescentes de cinco ciudades del Perú. Rev Peru Med Exp Salud Publica. 2015;32(3):499-503. 
En las zonas de endemicidad intermedia, (anticuerpos antihepatitis A positivo mayor al $50 \%$ a los 15 años), la edad media de infección es más alta $y$, paradójicamente, los casos, los brotes notificados y los síntomas asociados a la enfermedad son mayores, esto es debido a que circulan altas concentraciones virales en una población susceptible, lo cual lleva a que la morbilidad causada por hepatitis A sea un problema en incremento en las poblaciones en transición, de alta a intermedia endemicidad ${ }^{(4)}$. En las zonas de baja endemicidad, la seroprevalencia menor del $50 \%$ a la edad de 15 años.

Los estudios que evalúan la frecuencia de la hepatitis A son necesarios con el fin de establecer el patrón de endemicidad local y regional. En Perú vienen ocurriendo cambios socioeconómicos y ambientales que pueden estar influenciando en este patrón. Se han realizado trabajos que evalúan la frecuencia de hepatitis $A$ en algunos grupos poblacionales, sin embargo, a partir de estos no se puede determinar el patrón de endemicidad por grupos etarios y niveles socioeconómicos ni la diferencia entre las regiones del país ${ }^{(7-9)}$; por lo que el objetivo del presente estudio fue determinar la frecuencia de hepatitis $A$, en niños y adolescentes de 1 a 15 años de edad de cinco ciudades del Perú.

\section{EL ESTUDIO}

Se realizó un estudio transversal descriptivo entre los años 2010 al 2013. Se midieron anticuerpos totales antihepatitis A, en el suero de 1721 niños de 1 a 15 años.

El muestreo fue por conveniencia. A los niños que cumplían con los criterios de inclusión se les tomó suero en forma consecutiva, en cada una de las ciudades estudiadas en centros de atención, hospitales y clínicas de las ciudades de Arequipa, Cajamarca, Iquitos, Lima y Piura, hasta obtener el número necesario por cada subgrupo etario y socioeconómico.

Las cinco ciudades elegidas son representativas de diferentes zonas geográficas del Perú. Lima, es la ciudad más grande del país, con una población de 9 millones de habitantes, con el mejor acceso a educación y salud. Iquitos, la ciudad más grande de la Amazonía peruana, con 500000 habitantes, tiene una economía basada en el turismo, la industria extractiva y un nivel de pobreza del $36 \%$. Piura, con 430000 habitantes, situada en la costa norte de Perú, con una economía basada en la minería, pesquería y petróleo, y un nivel de pobreza del 35\%. Arequipa, con 910000 habitantes, la ciudad más grande del sur del Perú, dedicada a la agricultura, construcción, manufactura y minería y un nivel de pobreza de 9\%, y Cajamarca con 283700 habitantes, la ciudad más grande de la sierra norte, con una economía basada en el turismo, minería y ganadería y un nivel de pobreza del $39 \%$.

Se estableció el tamaño de la muestra a estudiar en cada ciudad, basados en datos publicados de prevalencia de hepatitis A en la ciudad de Lima. El tamaño de la muestra fue definido estableciendo los siguientes grupos etarios: 1 a 4 años, 5 a 9 años y 10 a 15 años. Los datos de población fueron tomados teniendo en cuenta los datos de población según edad y sexo por ciudad publicados por el INEI. La clasificación del nivel socioeconómico se hizo utilizando un cuestionario elaborado por la Asociación Peruana de Empresas de Investigación de Mercados (APEIM) lo que permitió identificar cinco niveles: A (alto/medio alto), B (medio), C (bajo superior), $D$ (bajo inferior) y $E$ (marginal). Para efectos del análisis, se agruparon los niveles $A$ y $B$.

Se excluyeron niños que hayan recibido la vacuna de hepatitis $A$, niños con enfermedad evolutiva severa, deficiencia inmunológica conocida (inclusive infección por HIV), administración previa de inmunoglobulinas o productos derivados de la sangre durante los últimos 6 meses

Las muestras de suero obtenidas fueron almacenadas en crioviales estériles de $2 \mathrm{~mL}$ a temperatura de 2 a $8^{\circ} \mathrm{C}$ en cada centro participante y periódicamente se enviaron al Laboratorio Central de Suiza Lab de Lima. Se midieron anticuerpos totales para hepatitis A (IgG $+\lg M)$, Las determinaciones se realizaron mediante el método de quimioluminiscencia para la detección de los anticuerpos totales frente al virus de la hepatitis $A$ (antiVHA) en suero. La preparación y procesamiento de las muestras, así como la interpretación de los resultados se realizaron teniendo en cuenta las instrucciones del fabricante.

La información se registró en una ficha de datos elaborada y tuvieron doble verificación. Se confeccionaron tablas de frecuencia, se calculó el chi cuadrado. Se consideró significativo un valor de p menor de 0,05 y se calculó su intervalo de confianza del 95\%. El análisis se realizó con el programa STATA versión 11.0.

Los pacientes fueron enrolados previa firma del consentimiento informado por parte de sus padres. La base de datos así como las fichas de recolección solo estuvo al alcance de los investigadores. Se guardó la confidencialidad de los datos. Los investigadores, conocían las implicancias éticas del estudio, y estos estaban claramente descritos en el protocolo de investigación. El estudio fue aprobado por el Comité de Ética de la Universidad Peruana Cayetano Heredia. 


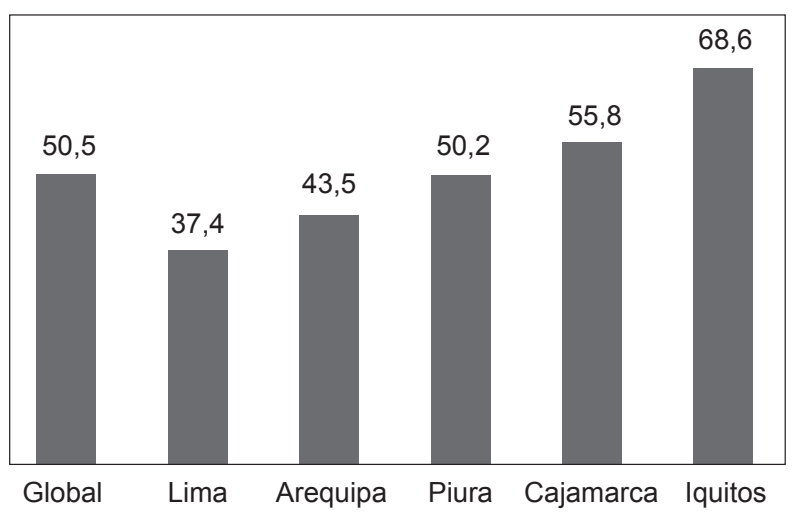

Figura 1. Frecuencia de serología positiva para hepatitis $A$ global y en cinco ciudades del Perú

\section{RESULTADOS}

Elresultadoglobal deserologíapositivaen lascincociudades fue de 50,5\% (IC 95\%: 48,1-52,9), correspondiendo a Lima 37,4\% (IC 95\%: 35,1-39,8), Arequipa 43,5\% (IC 95\%: 38,7-50,9), Piura 50,2\% (IC 95\%: 45,5- 54,9), Cajamarca 55,8\% (IC 95\%: 49,8-61,7) e Iquitos 68,7\% (IC 95\%: 63,6$73,4)$ (Figura 1). La población de lquitos tuvo 4,5 veces más frecuencia de serología positiva que la población de Lima; 2,8 veces más que Arequipa; 2,2 veces más que Piura y 1,7 más que Arequipa (Tabla 1 ).

Al analizar por niveles socioeconómicos (NSE) la frecuencia de serología positiva para hepatitis A fue inversamente proporcional al NSE: $\mathrm{AB}(33,1 \%), \mathrm{C}(47,1 \%)$, $D(53,1 \%)$ y $E(64,6 \%)$ (Figura 2$)$. Comparando el nivel $A B$ con el $E$ se encontró, que el nivel $E$ tiene 3,1 veces más frecuencia de serología positiva $(p=0,001)$. Al comparar

Tabla 1. Serología de hepatitis A según relación entre departamentos y niveles socioeconómicos

\begin{tabular}{lccc}
\hline & OR & (IC 95\%) & Valor $\boldsymbol{p}$ \\
\hline Departamentos & & & \\
Iquitos / Lima & 4,5 & $(3,2-6,2)$ & $<0,001$ \\
Iquitos / Arequipa & 2,8 & $(2,0-3,9)$ & $<0,001$ \\
Cajamarca / Lima & 2,5 & $(1,8-3,6$ & $<0,001$ \\
Iquitos / Piura & 2,1 & $(1,6-2,9)$ & $<0,001$ \\
Piura / Lima & 2,0 & $(1,5-2,8)$ & $<0,001$ \\
Iquitos / Cajamarca & 1,7 & $(1,2-2,4)$ & 0,008 \\
Cajamarca / Arequipa & 1,6 & $(1,1-2,3)$ & 0,003 \\
Arequipa / Lima & 1,5 & $(1,1-2,2)$ & 0,005 \\
Piura / Arequipa & 1,3 & $(0,9-1,7)$ & 0,07 \\
Niveles socioeconómicos & & & \\
E / AB & 3,1 & $(2,2-4,2)$ & 0,001 \\
C / AB & 1,5 & $(1,1-2,0)$ & 0,001 \\
E / C & 2,0 & $(1,5-2,7)$ & 0,003 \\
E / D & 1,5 & $(1,2-2,1)$ & 0,001 \\
D / C & 1,2 & $(0,9-1,6)$ & 0,050 \\
D / AB & 1,9 & $(1,4-2,5)$ & 0,001 \\
\hline
\end{tabular}

A (alto/medio alto), B (medio), C (bajo superior), D (bajo inferior) y E (marginal)

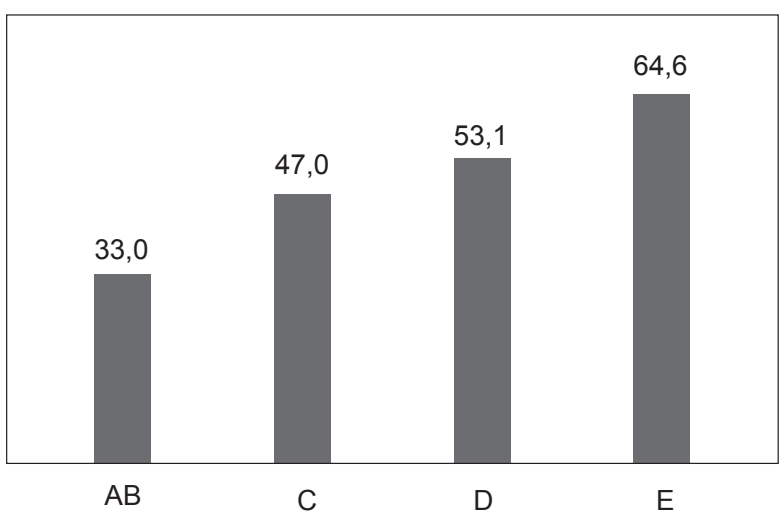

Figura 2. Frecuencia de serología positiva para hepatitis $A$ según nivel socioeconómico

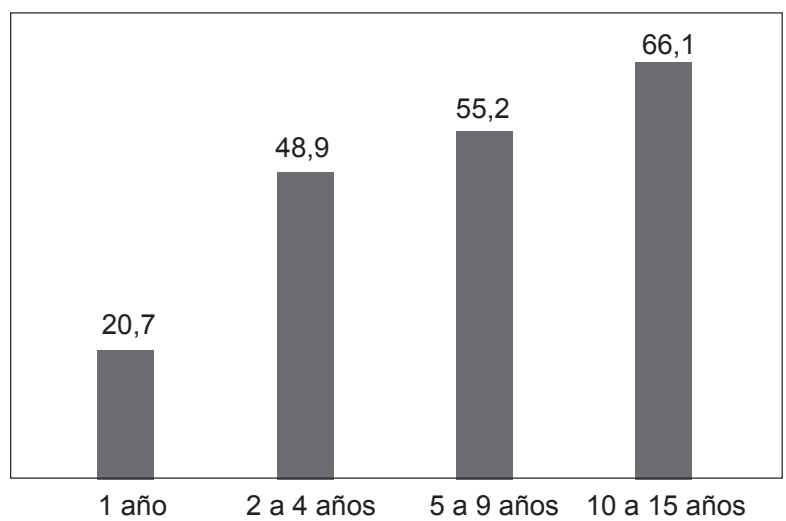

Figura 3. Frecuencia de serología positiva para hepatitis $A$ según grupo etario

el nivel socioeconómico $E$ con los otros niveles también observamos que la probabilidad de serología positiva siguió siendo 1,5 veces más frecuente que en el nivel $D$ y 2 veces más frecuente que en el nivel C (Tabla 1).

La frecuencia de serología positiva encontrada fue directamente proporcional a la edad, siendo menor en los niños de 1 a 2 años con $20,7 \%$ y mayor en los niños de 10 a 15 años con 66,1\% de infección (Figura 3). La serología positiva fue 3,9 veces más frecuente en el grupo de 10 a 15 años comparado la con los niños de 1 a 4 años $(p=0,001)$.

\section{DISCUSIÓN}

En el presente estudio se encontró que los más infectados por hepatitis A fueron los niños de la ciudad de lquitos, sobretodo si lo comparamos con Lima. Esta diferencia podría explicarse por las deficientes condiciones de salubridad de la ciudad de lquitos, así como por la diferencia de procedencia de las personas estudiadas de cada lugar ${ }^{(10,11)}$. 
En el Perú hay pocos datos sobre la prevalencia de anticuerpos contra el VHA en la población infantil. Un estudio realizado entre 1983 y 1984 en el que se incluyeron 811 niños de la costa y selva se encontró que el $82 \%$ de los niños tuvieron anticuerpos positivos anti-VHA. Asimismo, que el $30 \%$ de los niños de un año tenían serología positiva, incrementándose hasta el $100 \%$ a los ocho años de edad ${ }^{(9)}$.

En Lima, el año 1986 Kilpatrick reportó que los niños pertenecientes a los estratos socioeconómicos C y D tenían $98 \%$ de seropositividad para el VHA a los 9 años de edad y de $85,7 \%$ en el grupo de 5 a 9 años. Posteriormente, en el año 2000 Vildósola et al. en un estudio en el que incluyeron 859 personas entre 1 y 39 años, reportaron una seropositividad de $46,3 \%$ en menores de 14 años, en este grupo también se reportaron diferencias según NSE así, en el nivel $\mathrm{A}$ y $\mathrm{B}$, el 35,6\% tuvo serología positiva, y en el nivel C y D el $55,2 \%$.

Tal como se reporta en otras publicaciones a lo largo de los años, se evidencia una disminución de la serología positiva para infección por hepatitis A. Sin embargo, estos trabajos se realizaron en Lima y no se contaba con datos suficientes para determinar el patrón de endemicidad, de las diferentes regiones de Perú (7-9). Según la OMS, se pueden distinguir zonas geográficas de alta, media y baja endemicidad de infección por VHA.

Dado a que en Perú han venido sucediendo cambios económicos y sociodemográficos importantes, conocer el patrón de endemicidad del VHA es importante para definir políticas de salud pública, tenemos por ejemplo que la vacunación universal en la población infantil, en las poblaciones con alta endemicidad, no es costo beneficio, en cambio, sí lo es en las ciudades con prevalencia intermedia, mientras que en las ciudades con prevalencia baja el costo beneficio es mayor cuando la vacunación se dirige a grupos de riesgo ${ }^{(12-15)}$.

Al evaluar la serología positiva para hepatitis $A$, en los diferentes grupos etarios y ciudades, se encontró serología positiva en el $66 \%$ de sueros obtenidos entre niños de 10 y 15 años. Estos hallazgos nos colocan en un patrón de endemicidad intermedia, según definiciones de la OMS. En las ciudades estudiadas, el $20,7 \%$ de los niños de 1 a 2 años tuvieron serología positiva, variando entre $10,7 \%$ para Lima y $32 \%$ para Iquitos. Este dato es importante puesto que la vacunación en niños debería ser a partir del año de edad (edad mínima de vacunación) y no a los dos años, como se recomienda en otros países.

En cuanto a la diferencia regional, en el grupo etario de 1 a 4 años se observó que las ciudades menos afectadas fueron Lima y Piura, mientras que las más afectadas fueron Cajamarca e Iquitos. Esta diferencia según ciudad se mantuvo en los demás grupos etarios. Estos hallazgos podrían reflejar mejores condiciones de salubridad y educación en las primeras ciudades, especialmente en la ciudad de Lima.

La diferencia de serología positiva entre ciudades afectadas es mayor cuando comparamos los grupos etarios ya que en el grupo de 10 a 15 años la serología positiva, a excepción de Lima, tiende a ser parecida alrededor de $70 \%$, este hallazgo podría estar en relación a que la diferencia en las condiciones de salubridad que se han dado en los últimos años. Además, se puede señalar que en todas las ciudades, a medida que pasan los años, hay una mayor probabilidad de infección por hepatitis $A$, claramente podemos ver este hallazgo cuando comparamos el grupo etario de 10 a 15 años con los de 1 a 4 años, donde los de 10 a 15 años tuvieron 3,9 veces más serología positiva $p=0,001$. Es decir, se puede apreciar que la infección por hepatitis $A$ se está desplazando a grupo etarios mayores.

Este último hallazgo tiene importancia ya que se sabe que el rol de la edad es importante por la morbimortalidad de la hepatitis $A$, de hecho, el $50-90 \%$ de las infecciones adquiridas antes de los cinco años es asintomática; en cambio, el $70-90 \%$ de los adultos infectados tendrán síntomas ${ }^{(4)}$ y en cuanto a la letalidad tenemos que en menores de 5 años es 1,5 por mil, luego hay un descenso de tal manera que entre 5 y 14 años la mortalidad es de 0,004 por mil; entre 15 y 29 años es de 0,57 por mil; entre 30-49 años de 2,5 por mil, y en mayores de 49 años es 27 por mil infectados. Es decir, mientras más se desplaza la enfermedad a grupos etarios mayores, mayor será la mortalidad asociada. Por lo tanto, es posible que en los siguientes años si es que continúa esta tendencia, tengamos un patrón clínico diferente a lo que actualmente estamos acostumbrados.

Se encontró que a menor nivel socioeconómico mayor frecuencia de serología positiva, sin embargo, en forma interesante encontramos que si se analiza lquitos, esta tendencia es diferente, la serología positiva en los grupos $A B, C$ y $D$ es la misma, alrededor del $63,5 \%$; solo el grupo $\mathrm{E}$ tiene más $(79,3 \%)$, lo que indica que en esta ciudad la serología positiva es independiente del factor socioeconómico, lo cual se traduciría en una alta posibilidad de infectarse en cualquier área de la ciudad, básicamente por un problema de saneamiento ambiental observado en toda la ciudad. En Cajamarca también se observó que no hubo diferencia significativa entre los grupos $\mathrm{D}$ y $\mathrm{E}$.

Por los hallazgos podemos decir que en las ciudades estudiadas la infección por hepatitis A tuvo un patrón de endemicidad intermedia. Lo que podría significar que en los siguientes años se tenga una carga creciente de enfermedad por hepatitis A. En este escenario será de interés considerar 
como una medida de control la implementación de vacunación como una medida costo efectiva ${ }^{(16-19)}$.

Una limitación del estudio es que por su factibilidad es basado en una muestra por conveniencia y no aleatoria, por lo que podría tener un sesgo de selección. Sin embargo, nuestro estudio revela que los resultados pueden variar según el lugar de procedencia, por lo tanto, no necesariamente estos hallazgos se pueden extrapolar a todo el país. Al haber estudiado las principales ciudades de diferentes regiones, el conocimiento obtenido puede servir para entender las características de la distribución de la hepatitis $\mathrm{A}$.

En conclusión, la frecuencia de serología positiva para hepatitis $A$ en las ciudades estudiadas es un problema de salud púbica, en conjunto, tenemos un patrón de endemicidad intermedia donde Lima tiene la menor frecuencia e Iquitos la mayor. En general, la frecuencia de serología positiva se incrementó a mayor edad y en los de menor nivel socioeconómico.

Contribuciones de autoría: $\mathrm{RH}, \mathrm{EC}, \mathrm{CD}, \mathrm{MC}, \mathrm{EC}, \mathrm{RC}$ participaron en la concepción y diseño del trabajo, obtención de resultados, análisis de datos, redacción y revisión crítica del manuscrito, y aprobación de la versión final.

Fuentes de financiamiento: los kits utilizados para las determinaciones de laboratorio, materiales descartables y gastos operativos para la realización del estudio fueron subvencionados por el Laboratorio Sanofi Pasteur.

Conflictos de interés: los autores no tienen conflictos de interés en la publicación del artículo.

\section{REFERENCIAS BIBLIOGRÁFICAS}

1. Organización Mundial de la Salud [internet] 2014. [citado junio 2014]. Disponible en www.who.int/ mediacentre/囚FactSheet/fs328/es/

2. Vildósola H. Inmunización activa contra Hepatitis A. Rev Gastroenterol Perú. 2001;21(3):220-8.

3. Jacobsen KH, Wiersma ST. Hepatitis A virus seroprevalence by age and world region,1990 and 2005. Vaccine 2010;28(41):6653-7. doi: 10.1016/j. vaccine.2010.08.037.

4. Bell BP, Feistone SM. Vacuna antihepatitis A. En Plotkin S, Orenstein W, Picazo J. Vacunas. Madrid: ACINDES; 2007. p. 269-97.

5. Franco E. Meleleo C, Serino L, Sorbara D, Zaratti L. Hepatitis A: Epidemiology and prevention in developing countries. World J Hepatol. 2012;4(3):68-73. doi: 10.4254/wjh.v4.i3.68.

6. Lazcano-Ponce E, Conde-Gonzalez C, Rojas R, DeAntonio R, RomanoMazzotti L, Cervantes $\mathrm{Y}$, et al. Seroprevalence of hepatitis A virus in a cross-sectional study in Mexico: Implications for hepatitis A vaccination. Hum Vaccin Inmunother 2013;9(2):37581.

7. Kilpatrickl ME, Escamilla J. Hepatitis A in Peru: The role of children. Am J Epidemiology. 1986;124(1):111-3.

8. Mendez M, Arce M. Prevalencia de marcadores serológicos de hepatitis vírica en diversos grupos de población del Perú. B Ofic Sanit Panam. 1989;106(2):12738.
9. Vildósola H, Colichón A, Rubio MP, Weil J. Prevalencia de anticuerpos contra hepatitis A (Anti-HVA IgG) en una población de 1 a 39 años de Lima. Rev Gastroenterol Perú. 2000;20(2):141-5.

10. Fiore AE, Bell BP. Hepatitis A. En: Feigin RD, Cherry JD. Textbook of Pediatric Infectious Diseases, 6th ed. Philadelphia: W.B. Saunders Co; 2009. p. 2194-213.

11. CampagnaM1,SidduA,MeloniA,Basciu C, Ferrai L, Pettinau A, et al. Changing pattern of hepatitis a virus epidemiology in an area of high endemicity. Hepat Mon. 2012;12(6):382-5. doi: 10.5812/ hepatmon. 5940 .

12. Arza Fernández SB, Martínez de Cuéllar CM. Vacunación contra la hepatitis A: lineamientos técnicos y operativos para la vacunación contra el virus de la Hepatitis A [Internet]. Asunción: Viva Paraguay; 2013. [citado en abril del 2013]. Disponible en: http://www.mspbs.gov. py/pai/images/documentos/manuales/ manual_hepatitisa.pdf

13. Centers for Disease Control and Prevention; Fiore AE, Wasley A, Bell BP. Prevention of hepatitis A through active or passive immunization: recommendations of the Advisory Committee on Immunization Practices (ACIP). MMWR. 2006 May 19;55(RR-7):1-23.

14. American Academy of Pediatrics, Committee on Infectious Diseases. Hepatitis A vaccine recommendations. Pediatrics. 2007 Jul;120(1):189-99.

15. Organización Mundial de la Salud. Hepatitis A. Nota descriptiva N. ${ }^{\circ} 328$
[Internet]. Ginebra: OMS; 2015 [citado en Junio de 2014]. Disponible en: http:// www.who.int/mediacentre/factsheets/ fs328/es/

16. World Health Organization. Evidence based recommendations for use of hepatitis A vaccines in immunization services: Background paper for SAGE discussions [Internet]. Geneva: WHO; 2011 [citado el 17 de octubre del 2011]. Disponible en: http://www.who.int/ immunization/sage/meetings/2012/ april/1_HepABackground_17Oct_ final2_nov11.pdf

17. Cervio G, Trentadue J, D’Agostino D, Luque C, Giorgi M, Armoni J, et al. Decline in HAV associated fulminant hepatic failure and liver transplant in children in Argentina after the introduction of a universal hepatitis A Vaccination program. Hepat Med. 2011;3:99-106. doi: 10.2147/HMER. S22309.

18. Gentile A, Ramonet MD, Ciocca M. $\mathrm{La}$ introducción de la vacuna contra la hepatitis A en el Calendario Nacional de Vacunación: una nueva realidad. Arch Argent Pediatr. 2013;111(2):155-61.

19. Wasley A, Fiore A, Bell BP. Hepatitis A in the era of vaccination. Epidemiol Rev. 2006;28:101-11.

Correspondencia: Roger Hernández Díaz Teléfono: 998045048

Correo electrónico:rogerahd@gmail.com 